\title{
Modelado molecular de un ánodo de carbón activado como soporte de platino por medio de cálculos DFT
}

\section{Molecularly modeling an activated carbon anode as a platinum support by means of DFT calculations}

\author{
VALENZUELA-HERMOCILLO, Ernesto $\dagger^{1 *}$, PACHECO-SÁNCHEZ- Juan ${ }^{2}$ y RIVAS-CASTRO, \\ Sofía ${ }^{1}$
}

${ }^{\text {I}}$ Tecnológico de Estudios Superiores de Villa Guerrero. Carrertera federal Toluca- Ixtapan de la Sal Km. 64, Col. La Finca, C.P. 51763, Villa Guerrero, México.

${ }^{2}$ Instituto Tecnológico de Toluca, C.P. 52149, Estado de México, México

ID $1^{\mathrm{er}}$ Autor: Ernesto, Valenzuela-Hermocillo / ORC ID: 0000-0002-1126-5835

ID $1^{\text {er }}$ Coautor: Juan, Pacheco-Sanchez / ORC ID: 0000-0002-9525-5473

ID $2^{\text {do }}$ Coautor: Sofía, Rivas-Castro / ORC ID: 0000-0001-9278-0793

DOI: $10.35429 / J C P E .2019 .19 .6 .31 .38$

Recibido 09 Abril, 2019; Aceptado 28 Junio, 2019

\begin{abstract}
Resumen
En este trabajo se presenta el modelamiento molecular de un ánodo de carbón activado como soporte de platino de una celda de combustible de membrana de intercambio protónico (PEMFC), en la cual interacciona platino $(\mathrm{Pt})$ como catalizador soportado en carbón activado (CA) con hidrógeno molecular $\left(\mathrm{H}_{2}\right)$ con el fin de romperlo o ionizarlo. Se analiza la adsorción que existe entre CA y Pt como átomo o como una estructura superficial FCC(cúbica centrada en las caras) mínima, para observar si existe alguna diferencia entre carbón activado y sin activar en la reacción Pt- $\mathrm{H}_{2}$. Usando cálculos cuánticos de la Teoría del Funcional de la Densidad (DFT), se investigó la adsorción y el efecto del carbón activado sobre platino, empleando distintas estructuras de carbón y de platino. Nuestros resultados muestran que los carbones activados proporcionan un soporte estable para los catalizadores de platino, teniendo en cuenta que el CA presenta un pozo de energía potencial menor para adsorber el platino (fisisorción), en cambio un Carbón sin activar presenta un pozo de energía potencial mayor (quimisorción).
\end{abstract}

DFT, Carbón Activado, Soporte de Platino

\begin{abstract}
In this paper we present the molecular modeling of an activated carbon anode as platinum support of a proton exchange membrane fuel cell (PEMFC), in which platinum $(\mathrm{Pt})$ interacts as a catalyst supported in activated carbon $(\mathrm{CA})$ with molecular hydrogen $\left(\mathrm{H}_{2}\right)$ in order to break it or ionize it. The adsorption between $\mathrm{CA}$ and $\mathrm{Pt}$ is analyzed as an atom or as a minimum FCC(face-centered cubic) surface structure, to observe if there is any difference between activated and non-activated carbon in the $\mathrm{Pt}-\mathrm{H}_{2}$ reaction. Using quantum calculations of the Density Functional Theory (DFT), the adsorption and the effect of activated carbon on platinum were investigated, using different carbon and platinum structures. Our results show that the activated carbons provide a stable support for the platinum catalysts, taking into account that the CA presents a well of lower potential energy to adsorb the platinum (physisorption), whereas an activated carbon has a potential energy well major (chemisorption).
\end{abstract}

DFT, Activated Carbon, Platinum Support

Citación: VALENZUELA-HERMOCILLO, Ernesto, PACHECO-SÁNCHEZ- Juan y RIVAS-CASTRO, Sofía. Modelado molecular de un ánodo de carbón activado como soporte de platino por medio de cálculos DFT. Revista de Energía Química y Física. 2019. 6-19: 31-38

\footnotetext{
* Correspondencia al Autor (Correo electrónico: neto_alex@hotmail.com)

$\dagger$ Investigador contribuyendo como primer Autor
} 


\section{Introducción}

El actual uso excesivo de combustibles fósiles ha ocasionado el aumento de las emisiones de contaminación ambiental, así como, la creciente demanda energética a nivel mundial, han provocado la necesidad de diseñar e investigar nuevas fuentes alternas para producir energía eléctrica de manera más eficiente y amigable con el medio ambiente (Appleby, A. J. 1996). Las celdas de combustible de hidrógeno tipo membrana de intercambio de protones(PEM), pueden convertir de manera eficiente la energía química en electricidad con el agua como único subproducto (Barreras, F., and Lozano, A. 2012).

Las fuentes de energía renovables en el futuro todavía pueden requerir que la energía se almacena como combustibles líquidos para el transporte. El carbón activado como soporte de catalizadores (platino, paldio, niquel) altamente selectivos, eficaces y robustos son de importancia, pueden llegar a cumplir con este desafío, y son un área activa de investigación (Valenzuela, A. 2016).

El carbón activado se caracteriza por su estructura porosa bien desarrollada, lo que le confiere una elevada superfice y capacidad de adsorcion (Stoeckli, F. 1990. Molina, S. M., Rodríguez, F., Caturla F., Sellés M. J. 1995). En las últimas décadas se han estudiado materiales carbonos que pueden ser utilizados tanto en sensores electroquímicos como soporte (electrodo) de catalizadores (platino) en celdas de combustible (Litster, S., Mclean, G. 2004., Kim, M., Park, J.N., Kim H., Song S., Lee W.H. 2006).

El objetivo de la investigación es analizar y comparar los datos teóricos con los experimentales de las reacciones que suceden en un electrodo de una celda de combustible y poder proponer nuevos materiales, como es el caso del carbón activado como soporte de catalizadores, para la construcción de las mismas celdas y que estas tengan una mayor eficiencia.

En los estudios teóricos de la interacción platino-carbón activado no son muy frecuentes, por lo tanto, tenemos que estudiar para entender claramente la mayor parte posible sus mecanismos de reacción. Esto se persigue con la intención de construir un electrodo de una celda de combustible.
Mediante el uso de la teoría funcional de la densidad (DFT) (Parr, R., Yang, W. 1989). Se analizará la adsorción de distintas estructuras de carbón y carbón activado sobre el platino. Para llegar a la energía de disociación entre dos átomos hay dos maneras. La fisisorción (adsorción física) se produce una interacción de van der Waals, estas son de largo alcance, pero son débiles. La fisisorción se puede medir registrando el incremento de temperatura de una muestra de capacidad calorífica conocida, siendo los valores normales de alrededor de $20 \mathrm{~kJ} / \mathrm{mol}$.

En la quimisorción (adsorción química) se produce una unión formando un enlace químico, normalmente covalente, teniendo a buscar posiciones que maximicen su número de coordinación con el sustrato. La quimisorción son mucho mayores que las de fisioadsorción, estando alrededor de los $200 \mathrm{~kJ} / \mathrm{mol}$ (Atkins P., Friedman R. 2005). La distancia entre los átomos es también mucho menor en la quimisorción que en la fisisorción. Las energías de fisisorción y quimisorción que se calcularon están presentes en el documento, y se compararán con los datos teóricos y experimentales.

\section{Metodología a desarrollar}

Investigamos las interacciones del carbón y carbón activado como soporte de átomos de platino, variando las estructuras de carbón (Anillo de 6 carbonos, anillo activado de 6 carbonos, Grafeno y Grafeno activado) a través del programa de estructura electrónica DFT propuesto por Delley (Delley, B., Ellis, D., Freeman, A. and Baerends, E. 1983). Se utilizó un funcional de tipo local para el potencial de intercambio y correlación, este funcional propuesto por Perdew-Wang (PWC) (Perdew, J.P. and Wang, Y. 1992) de la Aproximación de densidad local(LDA) (Von Barth, U.; Hedin, L. 1972), incluyendo la polarización de spin (Oliver, G. L.; Perdew, J. P. 1979).

Los sistemas en estudio se corrieron utilizando los cálculos de spin no restringidos, all electrón y un conjunto de base de tipo DND(doble numérical + d-DNP), el tamaño del conjunto de bases DND es comparables a los conjunto Gaussian 6-31G*, además se incluyen los cálculos de campo autoconsistente (SCF) (Atkins, P.W. 1999). 
Las modelaciones iniciaron con sistemas pequeños de átomos de platino $(\mathrm{Pt})$ y átomos de carbón (C) y se fue aumentando el numero de átomos C (Placa de grafeno) y a su vez los åtomos de Pt (Estructura FCC de platino) en el plano [1,1,1], encontrando las distancias de interacción y energías de equilibrio, y así construir las curvas de energía potencial y poder analizar el efecto que causa el carbón activado sobre el platino (la adsorción del sistema). Las estructuras que se emplearon fueron activadas con hidróxido de potasio $(\mathrm{KOH})$ para el anillo y cloruro de zinc $\left(\mathrm{Cl}_{2} \mathrm{Zn}\right)$ para la placa de grafeno (Rivas, S.F., Pacheco, J.H. 2016., PachecoSánchez, J. H., Zaragoza-Rivera, I. P. and Bravo-Ortega, A. A. 2016).

\section{Resultados}

El sistema de estudio se enfocó en analizar el efecto que causa el carbón activado sobre el platino, se utilizó carbón activado obtenido mediante activación química, los agentes activantes que se usaron para activar fueron $\mathrm{KOH}$ y $\mathrm{Cl}_{2} \mathrm{Zn}$, obtenido en simulaciones moleculares ( Rivas, S.F., Pacheco, J.H. 2016, Pacheco-Sánchez, J. H., Zaragoza-Rivera I. P. and Bravo-Ortega, A. A.. 2016). Como dato de partida para nuestra investigación se tiene que la energía experimental de interacción Pt-C es de $6.28 \mathrm{eV}$ (Atkins, P.W. 1999), lo que equivale a $144.82 \mathrm{Kcal} / \mathrm{mol}$. Por lo que con esta información y apoyándonos de la teoría de los funcionales de la densidad, se inicio la investigación buscando cual aproximación y que funcional se aproximara a este dato experimental.

\section{Interacción del sistema $\mathrm{C}_{6}$ y $\mathrm{C}_{6} \mathrm{~A}$ con un átomo de platino}

En las simulaciones de las interacciones $\mathrm{C}_{6} \mathrm{~A}-\mathrm{Pt}$ (Figura 1) y la $\mathrm{C}_{6}-\mathrm{Pt}$ (Figura 2), encontramos que las distancias interatómicas del $\mathrm{C}_{6}$ y $\mathrm{C}_{6} \mathrm{~A}$ son similares, ya que el $\mathrm{C}_{6}$ tiene sus enlaces de 1.331 $\AA$ y las del $\mathrm{C}_{6} \mathrm{~A}$ oscila entre los $1.333 \AA$ y 1.423 $\AA$.

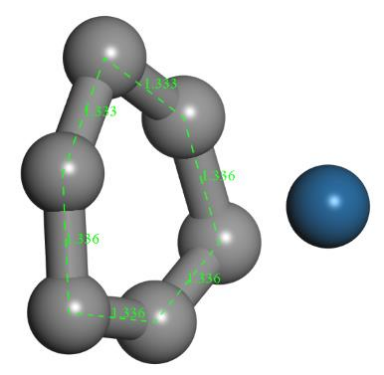

Figura 1 Optimización geométrica del sistema $\mathrm{C}_{6} \mathrm{~A}-\mathrm{Pt}$.

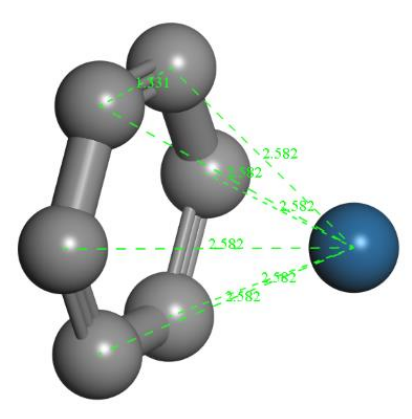

Figura 2 Optimización geométrica del sistema $\mathrm{C}_{6}-\mathrm{Pt}$.

En cuanto a la energía de equilibro encontramos que se necesita $-986.053 \mathrm{Kcal} / \mathrm{mol}$ para formar la molécula $\mathrm{C}_{6} \mathrm{~A}-\mathrm{Pt}$ y la molécula $\mathrm{C}_{6}$-Pt necesita $-983.141 \mathrm{Kcal} / \mathrm{mol}$, y la distancia de la molécula $\mathrm{C}_{6} \mathrm{~A}-\mathrm{Pt}$ es de $2.742 \AA$, la cual es mayor que la de la molécula $\mathrm{C}_{6}-\mathrm{Pt}$ que tiene $2.582 \AA$ y el tamaño del pozo de energía potencial es de $-25.182 \mathrm{Kcal} / \mathrm{mol}$ (Gráfico 2) para la molécula $\mathrm{C}_{6}-\mathrm{Pt}$ y de $\quad-19.511$ $\mathrm{Kcal} / \mathrm{mol}$ (Gráfico 1) para la molécula $\mathrm{C}_{6} \mathrm{~A}-\mathrm{Pt}$.

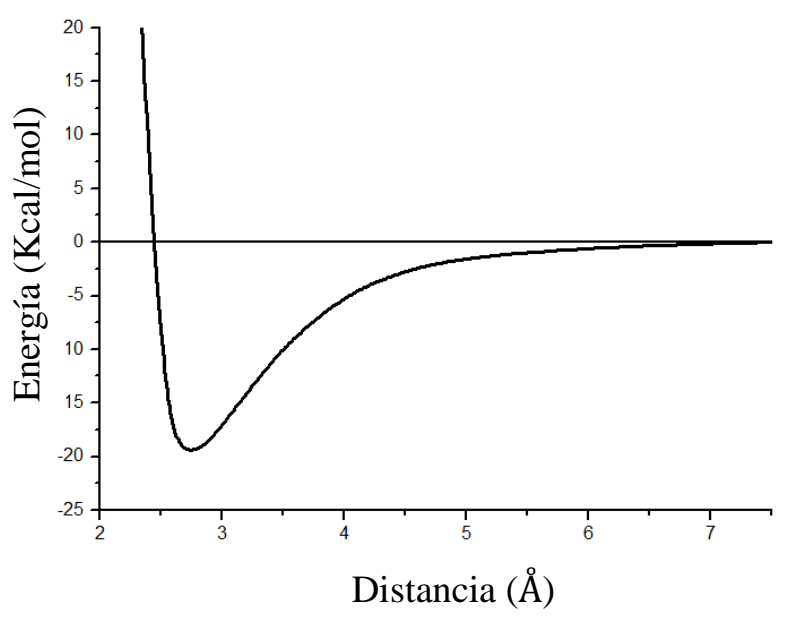

Gráfico 1 Curva de pozo de energía potencial del sistema $\mathrm{C}_{6} \mathrm{~A}-\mathrm{Pt}$.

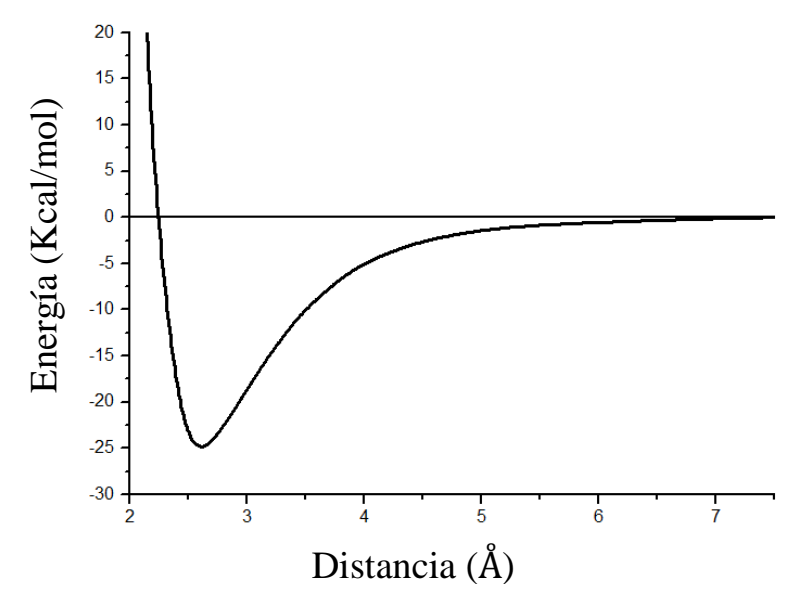

Gráfico 2 Curva de pozo de energía potencial del sistema $\mathrm{C}_{6} \mathrm{Pt}$. 
Por lo que nos damos cuenta que a pesar de que la energía y la distancia de la molécula $\mathrm{C}_{6} \mathrm{~A}-\mathrm{Pt}$ es más grande se tiene un tamaño de pozo de energía potencial menor que el de la molécula $\mathrm{C}_{6}-\mathrm{Pt}$, lo que nos indica que la adsorción del $\mathrm{C}_{6} \mathrm{~A}$ sobre $\mathrm{Pt}$ tiende a una fisisorción y $\mathrm{C}_{6}$-Pt tiende a una quimisorsión.

\section{Interacción perpendicular de la molécula de Hidrógeno y un átomo de platino}

Para encontrar la energía del hidrógeno se realizó la simulación del sistema $\mathrm{Pt}-\mathrm{H}_{2}$ para romper el enlace de la molécula de hidrógeno $\left(\mathrm{H}_{2}\right)$, para ello se colocó la molécula de hidrógeno y a un átomo de platino a una distancia de $1.335 \AA$ A del centro del átomo de platino a cada uno de los átomos de hidrógeno (H), con una distancia interatómica de la molécula de hidrógeno de 0.7 Å. (Figura 3 )

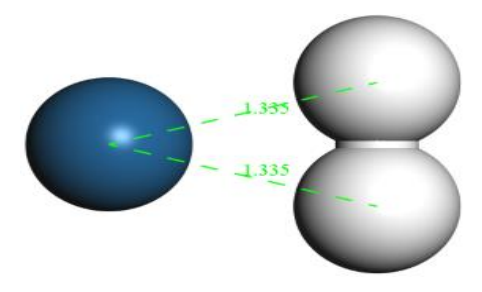

Figura 3 Sistema $\mathrm{Pt}-\mathrm{H}_{2}$.

Después de la optimización geométrica del sistema $\mathrm{Pt}-\mathrm{H}_{2}$ se encontró que el punto de equilibrio está a una distancia de $1.808 \AA$ y una energía de $-183.3 \mathrm{kcal} / \mathrm{mol}$ (Figura 4a). Se le realizó el cálculo de conectividad y se rompió el enlace de los átomos de hidrógeno y cada uno de los átomos de hidrógeno se enlazaron al átomo de platino (Figura 4b ).

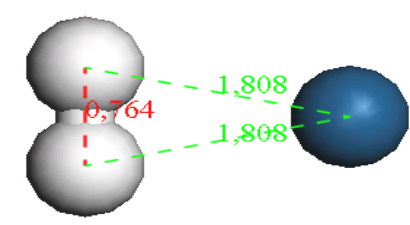

a)

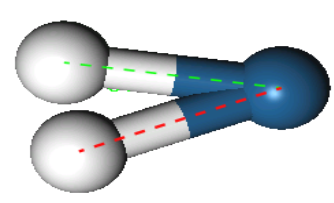

b)
Figura 4 a) Optimización geométrica del sistema $\mathrm{Pt}-\mathrm{H}_{2}$; b) Calculo de conectividad

Para obtener la energía que genera la molécula de hidrógeno al romper el enlace y tener dos átomos de hidrógeno, se calculó el pozo de energía potencial y para ello se fijo el átomo de platino y se fueron alejando y acercando los átomos de hidrógeno y se obtuvo un tamaño del pozo de $-22.751 \mathrm{kcal} / \mathrm{mol}$ (Gráfico 3) lo que equivale a $0.9865 \mathrm{eV}$.

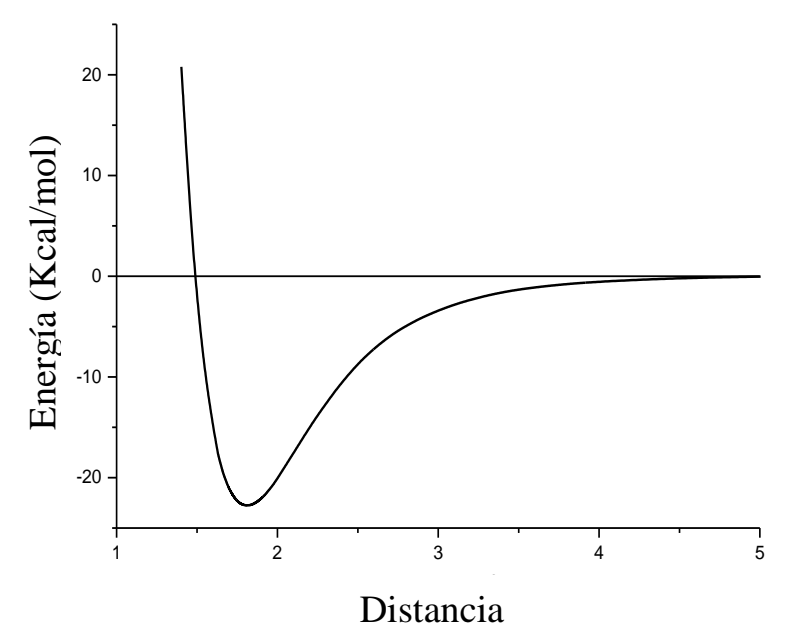

Gráfico 3 Curva de energía potencial del sistema $\mathrm{Pt}-\mathrm{H}_{2}$

Al compara el tamaño de pozo de la gráfico 3 con el valor experimental de la interacción $\mathrm{Pt}-\mathrm{H}_{2}$ se encontró que se aproxima, ya que el valor experimental es de 26.52 $\mathrm{Kcal} / \mathrm{mol}$ lo que equivale a $1.15 \mathrm{eV}$ (Emrick, R.M. 1982).

\section{Influencia del anillo de carbón en la reacción $\mathbf{P t H}_{2}$}

La influencia del $\mathrm{C}_{6}$ en la reacción Pt- $\mathrm{H}_{2}$ (Figura 5a ) se comparan los sistemas Pt- $\mathrm{H}_{2}$ y $\mathrm{C}_{6}-\mathrm{Pt}-\mathrm{H}_{2}$ (Figura 5b), para ello se calcularon los puntos de equilibrio y tamaño del pozo de energía potencial de ambos sistemas, la molécula de $\mathrm{PtH} 2$ se forma a una energía $\mathrm{E}_{\mathrm{eq}}=-183.3$ $\mathrm{Kcal} / \mathrm{mol}$ y una distancia de $1.808 \AA$ con un tamaño de pozo de $-22.751 \mathrm{kcal} / \mathrm{mol}$, y para formar la molécula de $\mathrm{C}_{6}-\mathrm{Pt}-\mathrm{H}_{2}$ se necesita una energía de $E_{\text {eq }}=-1126.490 \mathrm{Kcal} / \mathrm{mol}$ a una distancia $r_{e q}=1.827 \AA$ y el tamaño del pozo de energía potencial es de $-23.301 \mathrm{kcal} / \mathrm{mol}$ (Gráfico 4).

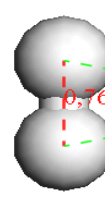

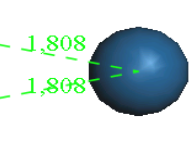

a)

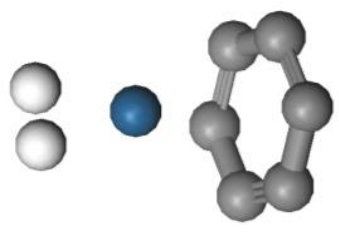

b)
Figura 5 a) Optimización geométrica del sistema $\mathrm{Pt}-\mathrm{H}_{2}$; b) Optimización geométrica del sistema $\mathrm{C}_{6}-\mathrm{Pt}-\mathrm{H}_{2}$

Dado que las energías del tamaño de pozo de estos sistemas son casi iguales, entonces se puede dice que el $\mathrm{C}_{6}$ además de fungir como un mero soporte también aporta propiedades catalíticas y la superficie de $\mathrm{C}_{6}$ no sufre modificaciones. 


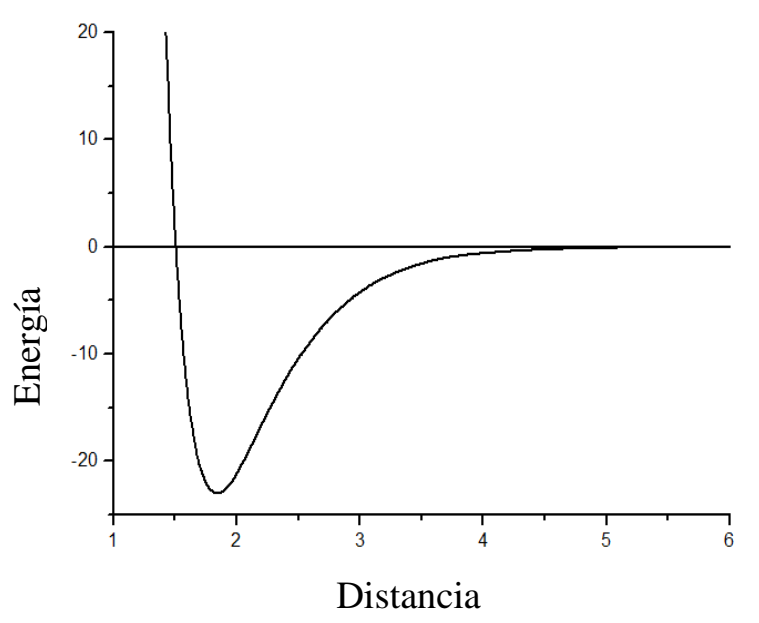

Gráfico 4 Curva de pozo de energía potencial del sistema $\mathrm{C}_{6}-\mathrm{Pt}-\mathrm{H}_{2}$.

Dado que las energías del tamaño de pozo de estos sistemas son casi iguales, entonces se puede dice que el $\mathrm{C}_{6}$ además de fungir como un mero soporte también aporta propiedades catalíticas y la superficie de $\mathrm{C}_{6}$ no sufre modificaciones.

\section{Interacción del sistema estructura cristalina FCC de platino soportada en una estructura grafeno y estructura cristalina fcc de platino soportada en una estructura grafeno activada}

En la simulación entre la estructura cristalina FCC de platino soportada en una estructura grafeno (Figura 6) y estructura cristalina FCC de platino en el plano $(1,1,1)$ (Arenas De, P. H. 1994). soportada en una estructura grafeno activada (Figura 7). Para ello se encontró que los puntos de equilibro de estas estructuras se encuentran a una distancia de $2.196 \AA$ y una energía de $-5814.346 \mathrm{kcal} / \mathrm{mol}$ y un pozo de potencial de $-217.674 \mathrm{Kcal} / \mathrm{mol}$ (Gráfico 5); y a $2.956 \AA$ de distancia y $-5741.73 \mathrm{kcal} / \mathrm{mol}$ de energía y un pozo de potencial de $101.181 \mathrm{Kcal} / \mathrm{mol}$ (Gráfico 6), respectivamente.

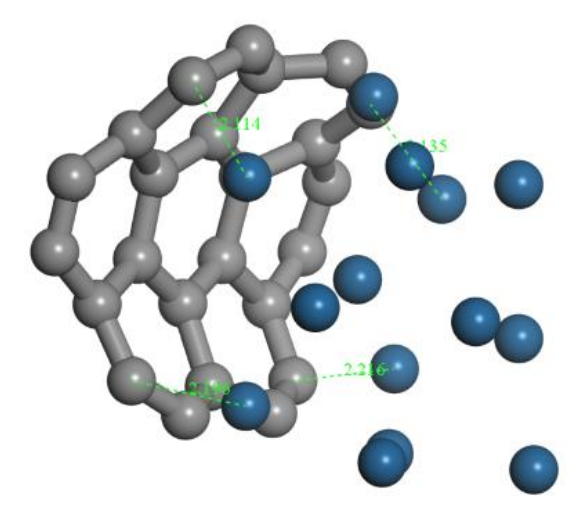

Figura 6 Optimización geométrica del arreglo estructura cristalina FCC de platino en el plano $[1,1,1]$ soportada en una estructura grafeno

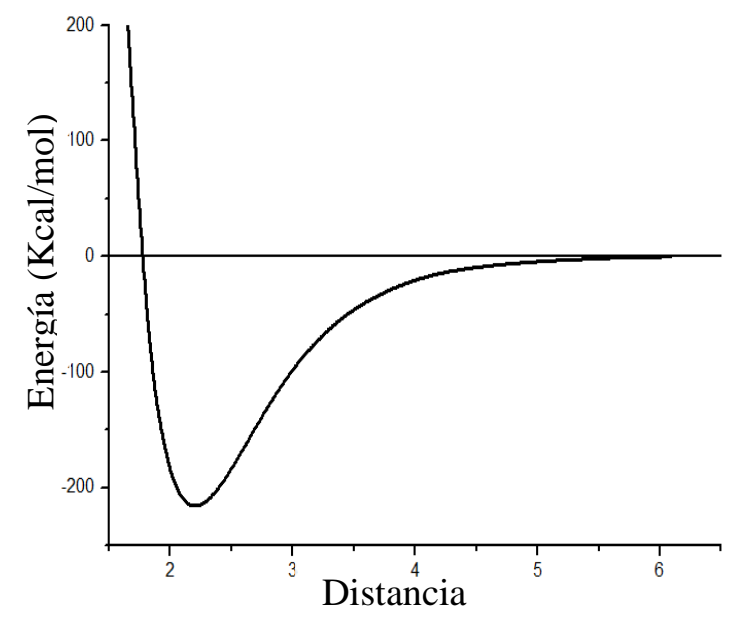

Gráfico 5 Curva de pozo de energía potencial del arreglo estructura cristalina FCC de platino en el plano $[1,1,1]$ soportada en una estructura grafeno.

Al hacer crecer el sistema llegamos a estos datos donde encontramos que se necesita menos energía para formar estructura cristalina FCC de platino soportada en una estructura grafeno activada, que la que se necesita de la estructura cristalina FCC de platino en el plano $[1,1,1]$ soportada en una estructura grafeno.

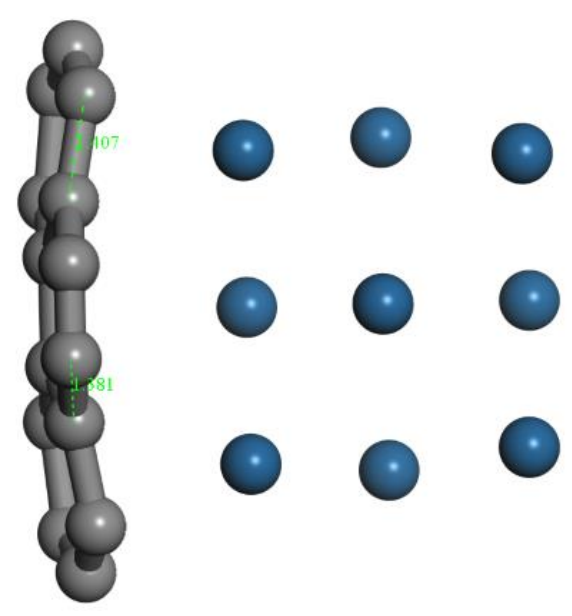

Figura 7 Optimización geometrica del arreglo estructura cristalina FCC de platino en el plano $[1,1,1]$ soportada en una estructura grafeno activado

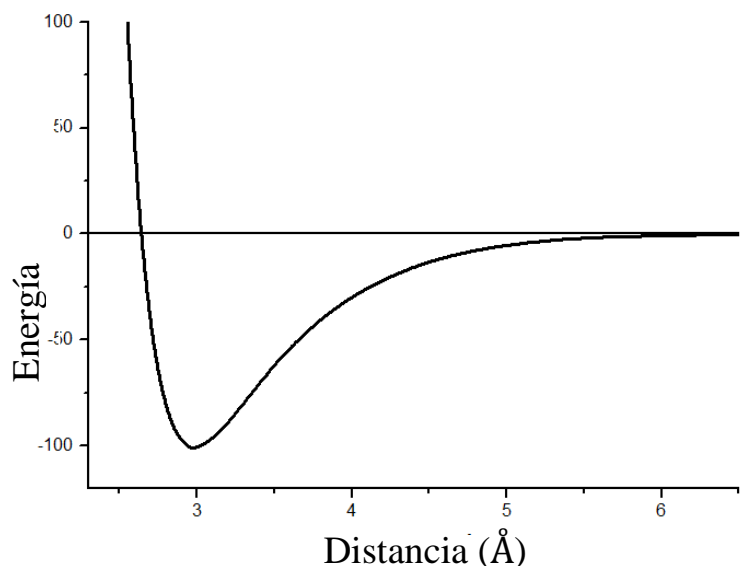

Gráfico 6 Curva de pozo de energía potencial del arreglo estructura cristalina FCC de platino en el plano $[1,1,1]$ soportada en una estructura grafeno activada. 
Encontramos que el pozo de energía potencial es $50 \%$ más pequeño en la estructura de grafeno activada y está no sufre modificaciones superficiales comparadas con las del grafeno sin activar. Así mismo la estructura FCC de platino se desordena en presencia de grafeno, mientras que la estructura fcc de platino en el plano $[1,1,1]$ en presencia del grafeno activado no sufre modificaciones estructurales relevantes.

\section{Interacción del sistema estructura cristalina fcc de platino soportada en grafeno con dos moléculas de hidrógeno}

La estructura cristalina FCC de platino en el plano [1,1,1] soportada en grafeno con dos moléculas de hidrógeno se comparó con el sistema Pt- $\mathrm{H}_{2}$. Para ello se calculó el punto de equilibrio de la estructura cristalina FCC de platino soportada en grafeno con dos moléculas de hidrógeno.

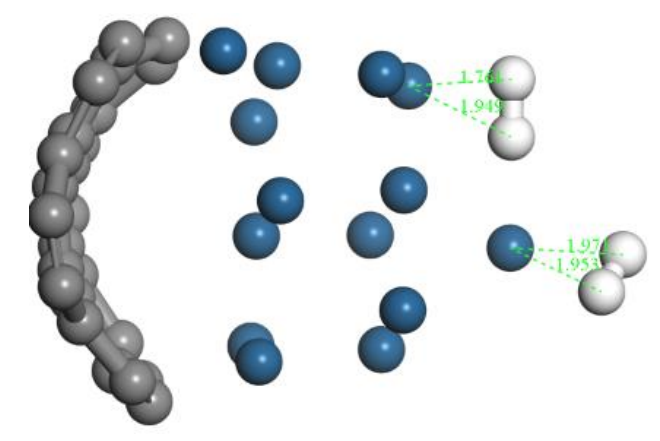

Figura 8 Optimización geométrica del arreglo estructura cristalina FCC de platino soportada en grafeno con dos moléculas de hidrógeno

Se encontró que tiene una energía de equilibrio de $-6069.151 \mathrm{kcal} / \mathrm{mol}$ (Figura 8), y una distancia de equilibrio de $2.196 \AA$ entre el grafeno y la estructura cristalina fcc de platino, y $1.876 \AA$ entre la estructura FCC de platino y una molécula de hidrógeno.

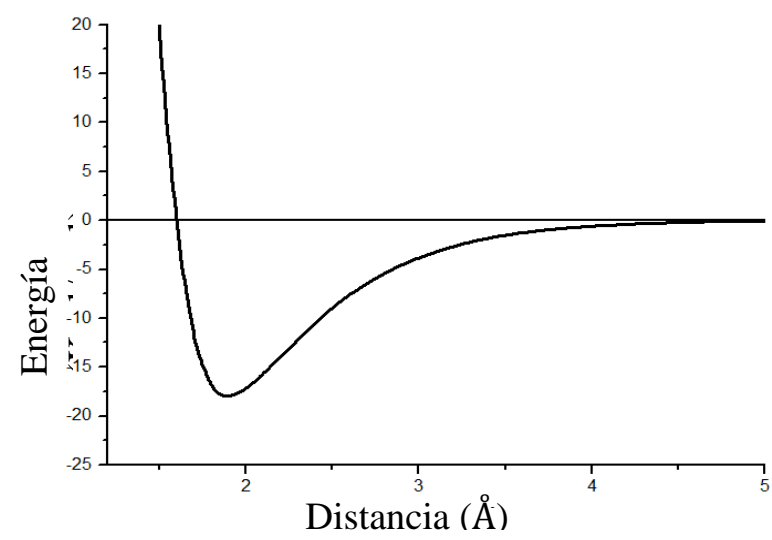

Gráfico 7.Curva de pozo de energía potencial del arreglo estructura cristalina fec de platino soportada en grafeno con dos moléculas de hidrógeno
Se calculo el tamaño del pozo de energía potencial variando(alejando y acercando) la distancia entre un átomo de platino de la estructura cristalina FCC de platino y una de las moléculas de hidrogeno y se tuvo que el tamaño de pozo de energía potencial (gráfico 7) es de $18.164 \mathrm{Kcal} / \mathrm{mol}$, que al compararlo con el pozo de energía potencial del sistema $\mathrm{Pt}-\mathrm{H}_{2}$ que es de $-22.751 \mathrm{kcal} / \mathrm{mol}$, por lo que se dice que la magnitud de; tamaño de pozo se reduce en un $20 \%$. Además el valor de $-18.164 \mathrm{Kcal} / \mathrm{mol}$ es igual a $0.78 \mathrm{eV}$ lo que es aproximado al que se obtiene de voltaje de salida en una monocelda de combustible real (Pacheco, J.H., García, L.A., Zaragoza, I.P. and Bravo, A. (2005).

\section{Conclusiones}

En este trabajo se ha estudiado la interacción entre el platino soportado en estructuras de carbón y carbón activado, utilizando la metodología, DFT con LDA/ PWC.

$\mathrm{El}$ sistema $\mathrm{C}_{6}-\mathrm{Pt}$ que tiene una magnitud de $-25.182 \mathrm{kcal} / \mathrm{mol}$ y corresponde al tamaño de pozo de energía potencial, tiende a una quimisorción y el sistema $\mathrm{C}_{6}$ - $\mathrm{APt}$ que tiene una magnitud de $-19.511 \mathrm{kcal} / \mathrm{mol}$ de tamaño de pozo de energía potencial tiende a una fisisorcion. Los materiales carbonosos $\mathrm{C}_{6}, \mathrm{C}_{6} \mathrm{~A}$, Grafeno y Grafeno Activado, influyen poco sobre la reacción $\mathrm{Pt}-\mathrm{H}_{2}$ ya que las magnitudes del tamaño de pozo de energía potencial son casi iguales.

Para las estructuras de grafeno y grafeno activado como soportes de estructuras cristalina de platino, se encuentra que ambas corresponden a una quimisorción por valores mayores a 50 $\mathrm{kcal} / \mathrm{mol}$ (Atkins, P.W. 1999). Las estructuras de grafeno activado no sufren modificaciones estructurales al soportar al platino en cambio el grafeno puro sufre un encorvamiento cóncavo hacia el platino.

Con la estructura cristalina fcc de platino soportado en grafeno se obtuvo que los valores de la simulación son cercanos a un $97.5 \%$ a los datos experimentales, ya que DFT obtuvimos $18.164 \mathrm{Kcal} / \mathrm{mol}$ equivalente a $0.78 \mathrm{eV}$ mientras que el experimental es $0.8 \mathrm{eV}$ (Pacheco, J.H., García, L.A., Zaragoza, I.P. and Bravo, A. 2005). 
Se determina que el carbón activado muestra una mejor superficie para la dispersión (adsorción) del platino con menor energía de disociación, pero aún es elevada para que se realice en condiciones de temperatura ambiente. Así mismo se llega a concluir que el carbón activado como soporte de catalizadores influye en las reacciones electroquímicas que suceden en el ánodo de una celdas de combustible tipo PEM.

De acuerdo con lo anterior mencionado el carbón activado como soporte de catalizadores es un excelente material para ser empleado en las celdas de combustible tipo PEM, así como también se podrá utilizar en otro tipos de celdas de combustible como son las celdas de combustible microbianas, ya que su capacidad de adsorción ayuda a que las reacciones se lleven acabo de mejor manera.

\section{Agradecimiento}

Se le agradece al Instituto Tecnológico de Toluca y al CONACyT por los apoyos brindados.

\section{Referencias}

Appleby, A. J. (1996). Fuel Cell Technology: Status and Future Prospects. Energy. V. 21, n. 7/8, p. 521-653.

Barreras, F. y Lozano, A. (2012). Hidrogeno. Pilas de combustible tipo PEM. Universidad de Zaragoza. Zaragoza, España.

Parr, R., Yang, W. (1989). "Density-Functional Theory of Atoms and Molecules". Published by Oxford University Press. New York. Pg.333

Delley, B.; Ellis, D. E.; Freeman, A. J.; Baerends, E. J.; (1983). "Energías de enlace y estructura electrónica de pequeñas partículas de cobre”. Post,D. Phys. Rev. B, 27, 2132.

Perdew, J. P. and Wang, Y. (1992). "Accurate and simple analytic representation of the electron-gas correlation energy". Phys. Rev. B 45 (23): 13244-13249.

Von Barth, U.; Hedin, L. (1972). "A local exchange-correlation potential for the spin polarized case". J. Phys. C: Solid State Phys. 5 (13): 1629-1642.
Oliver, G. L.; Perdew, J. P. (1979). "Spin-density gradient expansion for the kinetic energy". Phys. Rev. A 20 (2): 397-403.

Atkins, P.W. (1999). Química-física. Edición Omega, S.A. Universidad de Barcelona. Barcelona España.1018pp.

Arenas De, P. H. (1994). El estado solido y propiedades de los materiales. Universidad industrial de Santander, Bucaramanga.

Pacheco, J.H., García, L.A., Zaragoza, I.P. and Bravo, A. (2005). Fuel Cells Project Research in develope. Instituto Tecnológico y de Estudios Superiores Monterrey-Campus Estado de México.

Emrick, R.M. (1982). Journal of Physiscs F: Met Phys. 12. 1327.

EG\&G Services Parsons. (2000). Inc. Science Applications International Corporation. "Fuel Cell Handbook". U.S. Department of Energy Office of Fossil Energy National Energy Technology Laboratory. Pág. 1.1, 1.3, 1.4, 1.9, $1.10,1.11,1.12,1.13$

Litster S., Mclean G. (2004). PEM fuel cell electrodes. Journal of Power Sources 130, 6176.

Kim M., Park J. N., Kim H., Song S., Lee W.-H. (2006). The preparation of $\mathrm{Pt} / \mathrm{C}$ catalysts using various carbon materials for the cathode of PEMFC. Journal of Power Sources 163, 93-97.

Stoeckli, F. (1990). "Microporous carbons and their characterization: The present state of the art" Carbon 28,1-6.

Molina, S. M., Rodríguez, F., Caturla F., Sellés M. J. (1995). "Porosity in granular carbons activated with phosphoric acid" Carbon 33, 8, 1105-1113.

Rivas, S.F., Pacheco, J.H. (2016). Modelación del carbón activado mediante $\mathrm{KOH}$ e identificación de la estructura molecular en anillos. Enviado a publicación.

Pacheco-Sánchez, J. H., Zaragoza-Rivera, I. P. and Bravo-Ortega, A. A. (2016). Interaction of small carbon molecules and zinc dichloride DFT study. Enviado a publicación. 
Atkins, P., Friedman, R. (2005). Molecular Quantum Mechanics. Fourth Edition. Oxford University Press. New York. Unisted States. 573 pp.

Valenzuela, A. (2016). Construcción de un electrodo de carbón activado como soporte de platino por simulación molecular(tesis de posgrado). Instituto Tecnológico de Toluca, Metepec, Edo. de México. 